could not draw off the fibrin, that the advantage would be only temporary, as the fluid would re-accumulate, \&c., would become no arguments against the operation, provided we were perfectly sure of our diagnosis of a large amount of fluid.

\title{
TUMOR OF THE LARYNX REMOVED WITH SUCCESS.
}

[Read befure the Boston Society for Medical Improvement, and communicated for the Boston Medica] and Surgical Journal.]

By Samuer Cabot, M.D.

ThE patient, J. H. D., æt. 5 years, a healthy, well-developed boy for his age, was observed to have hoarseness, which began about a year since, and gradually became worse, the breathing showing signs of obstruction, without cough at first, but later some cough was occasionally present, which was attributed to bronchitis. About eight or nine months after the first appearance of the hoarseness, he had an attack of what was called pneumonia, after which the hoarseness and difficulty of breathing somewhat diminished, continuing better until recently, when it became rapidly worse, so as to destroy the power of articulation.

Saturday, Jan. 7th, 1865, I was called to the country to sce the child, who was said to be near death. I found him breathing with the greatest difficulty, livid, gasping, throwing himself about upon the bed, the head thrown back, \&c.; in short, all the appearance of imminent suffocation. On introducing my finger into the throat, the edges of the larynx felt thickened and rounded. The chest was resonant upon percussion throughout. Feeling that the only resource was tracheotomy, I immediately opened the trachea at as great a distance from the larynx as I conveniently could, and introduced a large double tube, with a fenestrum in the outer tube. The relief was immediate and complete. I advised that the boy should be taken to the Massachusetts General Hospital at the earliest practicable moment, that he might be under my immediate carc.

He came to Boston and entered the Hospital on the 10th, three days after the operation. He was put in a room well filled with steam, and put upon a nutritious diet, with internal use of iodide of potash, and frequent applications of a strong solution of tannic acid in glycerine to the throat and larynx.

On Jan. 12 th I made an examination of the larynx with the laryngoscope, but owing partly to the irritability of the fauces and partly to my own inexperience in the use of that instrument, my examination was far from satisfactory. The only fact learned by it was that the edges of the larynx were less thickened than I had supposed from my previous digital examination. Under the continued use of steam in the air, applications of tannin, chlorate of potash, alum, and nitrate of silver in solution and in the form of powder to the 
diseased part, and the internal use of iodide of potash, the patient apparently improved, and on Feb. 7th the steam was discontinued.

On Feb. 20th the patient was etherized, the tubes were removed from the trachea, and a large-sized metallic sound was passed through the larynx past the wound in the trachea, and withdrawn; still it was found that upon withdrawing the inner tube and stopping the orifice of the remaining one, the patient could not breathe through the larynx. He was sent to his home in the country, and the same treatment was ordered to be continued there.

On May 11th re-entered the Hospital, in order to have a different kind of tube substituted for the one he had been wearing. This tube was designed to pass up into the lower larynx, and to fit into another passing down in the direetion of the trachea; the part designed to pass up into the larynx was made with a hinge, allowing the upper portion, which was made of two parts, resembling the upper mandibles of a duck's bill, to shut together, so that it could be easily introduced, and then expanded when within the larynx. It was found upon trial, that though it could be easily introduced and secured in place, the instrument projected too far into the larynx, and caused so much irritation that it could not be retained.

Hoping that the obstruction, whatever its nature, might have so far diminished under treatment that the patient could get along without any tube, I decided to allow the opening in the trachea to close and watch the effect.

As the opening in the trachea contracted, the breathing became more and more labored; the child's health began to suffer, he could not sleep, and the breathing evidently could not be carried on through the larynx, so that upon the 14th, three days after the tubes had been left out, I was obliged to re-introduce them, which I did by enlarging the opening upwards, so as to put it at a point somewhat nearer the larynx than it had been previously. After this several attempts were made to substitute the duck-bill tube, the mandibles having been shortened, but though it was borne for several days on one occasion, yet it was found not to answer, and was finally abandoned. Finding that my own attempts at laryngoscopy had failed to give me much light upon the nature of the obstruction, I called in Dr. H. K. Oliver, our accomplished laryngoscopist, who, after a long and patient trial, extending over weeks, including as it did the education of a child of 6 years in the art of showing his larynx, succeeded at length in discovering that nearly the whole.larynx was filled up by what appeared to be a smooth, rounded tumor, growing from the whole posterior wall of the larynx, projecting forward, so that its anterior margin could not be seen, with no appearance of any fissure on either side of it, and precluding all hope of applying any wire-loop, or ecraseur, or other instrument for its removal. After consulting with Dr. Oliver, I scarified the tumor deeply with a curved knife, cutting on its convexity, and applied immediately VoL. LXXXIV.-No. 2 
a saturated solution of nitrate of silver; this was repeated several times, but with no permanent benefit. Having exhausted all other means, I finally determined to lay open the whole larynx, and thus to gain direct access to the tumor. Accordingly, on the 18th of November, the patient having been etherized, the tube was withdrawn from the trachca, the skin and soft parts cut through, from a point about an inch above the upper margin of the larynx to the opening in the trachea. Then a bent, grooved director was carried through the opening in the trachea up and out through the opening above the larynx, being held carefully to correspond to the line of junction between the thyroid cartilages; a knife was pushed up along the groove in the director, dividing all the parts above it, thus splitting the larynx its whole length; the sides were then pulled apart by vulsella, and held thus by aids. But little blood was lost, and on sponging out the blood the tumor was seen, occupying the posterior internal surface of the larynx, covering it completely from side to side, and almost entirely from top to bottom, and projecting forward so as to almost touch the anterior surfaces. The appearance and character of the tumor was quite different from what I had been led to expect from the appearances presented by the laryngoscope. Instead of a smooth, rounded, solid mass, such as I expected to find, and which I had hoped to seize with forceps and to dissect out with a knife, I found a mass resembling half a raspberry, with papillæ more pronounced and distinct; in short, a columnar, epithelial, wart-like growth, very friable, giving no hold to forceps, and breaking with the least touch. This I snipped off with curved scissors, after which I removed two firmer masses from the fossæ, and then having dried the surface, I rubbed it over very thoroughly with solid nitrate of silver. I then secured the thyroid cartilages together with a single stitch, and brought the skin together with sutures down to the margin of the opening in the trachea, into which I again introduced the tubes. The next day, the boy was up and playing with his toys; he said his throat felt sore, but made little complaint of it.

Nov. 23d, five days after the operation, he could bear to have the outer opening of the tube stopped, and could breathe with but little difficulty through his larynx. He was sent home to get the wound healed and the soreness removed, then to return and have the tubes taken out.

His wound soon healed and the soreness disappeared, and his mother, who had been directed to stop the tube frequently in order to accustom him to use his larynx which had been disused for nearly a year, reports that he often goes with a cork in the tube from the time he gets out of bed in the morning until he goes to bed at night, and without the slightest inconvenience.

Soon after this report was made by the mother, the boy took a severe cold, with cough, profuse mucous expectoration, \&c.; this attack kept him at home until Jan. 2d, 1866, when he re-entered the 
Hospital, and although he had not recovered from the cold, I removed the tube from the trachea. The orifice closed in about a week, his breathing becoming easier day by day, and voice gradually returning, so that he could speak in a loud whisper, could whistle, and blow with a strong and prolonged current. Before he went home, Dr. Oliver examined his larynx with the laryngoscope, and found it apparently free from disease, and with an opening of the natural size.

The microscopic examination of the tumor was made by two gentlemen, experts in microscopy, to one of whom was committed one of the firm masses removed from the fossæ, and was reported by him to consist of glandular and fibrous tissue; to the other, a part of the wart-like growth was given, and he reported that it consisted of epithelial and fibrous tissue.

About a week after this report was read to the Society, I was sent for to see the child, as he was very ill, with cough and difficulty of breathing, supposed to be pneumonia. When I saw him, I found that the pneumonic symptoms had disappeared; he had slight cough and some expectoration, but the breathing was labored, with a tracheal sound, and the passage of air through the trachea was not sufficiently free to support life, though there were no signs of imminent suffocation. Accordingly, I cut down in the old cicatrix and opened the trachea, which appeared much narrowed at this point, and covered with pediculated granulations, some of which were cut off by my knife, and some torn off by the tube on inserting it; they resembled very closely the peculiar growths seen in a case of disease of the larynx following caustic ammonia, and of which the tongue and larynx were shown by Dr. H. K. Oliver on the same evening that my paper was read.

I think that the present obstruction is wholly at the point in the trachea where the tube was so long worn, and is caused partly by cicatricial contraction and partly by the pediculated outgrowths of which I have spoken, and that this condition of things is mostly due to the long presence of the tube; moreover, that if the larynx had been opened at the first and the tumor excised then, the result would have been the perfect success which I had supposed I had reached.

\section{CHOLERA.}

Querieb bx a Practical Man (Suggested dy the rast No. op the Journat).

[Communicated for the Boston Medical and Surglcal Journal.]

IF "the disease cannot be communicated from one person to another even under circumstances of the greatest intimacy," how can it be conveyed by the clothing simply? If "the disease travels no faster than men travel," will keeping all men at home put an end 